\title{
8 The Touched Self: Psychological and Philosophical Perspectives on Proximal Intersubjectivity and the Self
}

\author{
Anna Ciaunica and Aikaterini Fotopoulou
}

\section{Introduction: The Mentalization of the Body and Minimal Selfhood}

Whenever I perceive something or feel an emotion, these perceptions and feelings are somehow given to me as mine. The idea that our everyday experiences are characterized by a prereflective sense of self, referred to as the "minimal" self, has been highlighted by a longstanding phenomenological tradition (Husserl, Sartre, Merleau-Ponty), as well as more recent authors (Gallagher 2000; Metzinger 2003; Zahavi 2005; Hohwy 2007; Blanke and Metzinger 2009; Blanke 2012). There is wide agreement about the importance of examining the bodily foundations of such prereflective forms of self-awareness, in the sense that one needs to view the mind as a support system that facilitates the functioning of the body and not the other way round. Crucially, bodily self-awareness is not an awareness of the body in passive isolation from the physical and social world. Indeed, both classic phenomenologists such as Husserl and Merleau-Ponty and, more recently, researchers working within the embodied and enactive cognition paradigm (Varela, Thompson, and Rosch 1991) insist on the idea that prereflective self-awareness ought to be understood primarily by taking into account the larger brain-body-environment dynamics (De Jaegher and Di Paolo 2007; Menary 2007). This emphasis has also been adopted by influential recent models of brain function in theoretical neuroscience (Friston 2010; see also Clark 2013), as we briefly outline later. The development of the mind, and selfhood more specifically, can therefore be viewed as the consequence of embodiment within its environment.

The question of what, if anything, makes the "self" a unifying phenomenon has attracted a considerable number of empirical studies and theoretical accounts. A detailed review of the literature dedicated to clarifying the notion of the self lies beyond the scope of this chapter. Rather, for our limited purposes here, it is important to note that despite disagreements on crucial questions about whether there is a self, to what degree it is prereflective, and what exactly constitutes this prereflective sense of self, most of the contemporary accounts share the important assumption that minimal selfhood is not to be conceived as a static internal snapshot of some mysterious substance called the "self." Instead, minimal selfhood is 
conceived as an ongoing process of tracking and controlling bodily properties as a whole (Blanke and Metzinger 2009). If this is so, then one of the main challenges for both theoretical and empirical accounts of minimal forms of self-awareness consists in characterizing the nature of the relational components of selfhood by taking into account the role of worldly engagements in shaping its different facets. Indeed, while there is wide agreement over the idea that prereflective self-awareness is a dynamic and more primitive form of awareness, it is still unclear whether the "ongoing" dynamic and "primitive" aspects refer exclusively to a selfcentered continuity or encompass self-other relatedness, as well. For example, Zahavi (2014, 2015a, 2015b) has recently drawn a careful analysis of minimal forms of selfhood and selfother relatedness by distinguishing between (1) "experiential minimalism" (EM) and (2) "social constructivism" (SC), which can be seen as two opposing poles of the debate (Zahavi 2015a). On the one hand, experiential minimalism claims that our experiential life is characterized by a prereflective sense of self or mineness that can and should be understood without any contrasting others. On the other hand, according to social constructivism, the minimal self is not innate but a later socioculturally determined acquisition, emerging in the process of social exchanges and mutual interactions. ${ }^{1}$

Against this background, we aim to argue in favor of a reconceptualization of minimal selfhood that transcends such debates and instead traces the relational origins of the self to fundamental principles and regularities of the human embodied condition, which includes social, embodied interactions and practices. Specifically, our position is motivated by the following five theoretical and empirical observations: (1) The progressive integration and organization of sensory and motor signals constitute the foundations of the minimal self, a process that we have elsewhere named "mentalization" of the body (Fotopoulou 2015). (2) Minimal selfhood is best understood by a conceptualization that takes into account all sensory and motor modalities, along with their distinct properties and rules of integration, instead of relying mostly on a "detached" visuospatial model of perceptual experience, and by extension a model of "detached" social understanding. (3) Crucially, as some of these sensorimotor modalities are specialized to respond to experiences both "within" and "on" the physical boundaries of the body (e.g., the skin), an experiencing subject is not primarily understood as being "here" and facing a perceptual object or subject "there," that is, in a separate physical location. (4) Instead, interactions with other people are motivated and constrained by the same principles that govern the "mentalization" of sensorimotor signals in the singular individual, and hence the mentalization of one's body includes any body in physical proximity and interaction. (5) Finally, given the premature birth and social dependency of humans in early infancy, there is a "homeostatically necessary," genetically

1. For example, W. Prinz (2012) argues that the self is essentially a social and cultural construct, and he emphasizes the socially constructed character of phenomenal consciousness. H.-B. Schmid holds the view that what is shared precedes the self-other distinction (Schmid 2005, 145, 149, quoted in Zahavi 2015a, 157; Schmid 2014). 
prescribed, and culturally enriched plethora of such embodied "proximal" experiences and interactions. Collectively, such experiences of proximal intersubjectivity "sculpt" the mentalization process and hence the constitution of the self, including the progressive sophistication of mental distinctions between "subject-object," "here-there" and "goodbad." We unpack these points by focusing predominately on the domain of touch as a paradigmatic example of proximal intersubjectivity.

Before proceeding, a few brief conceptual clarifications are needed. What is prereflective self-awareness? Philosophers usually start with the intuitive idea of reflecting on our inner experiences: for example, I can introspect what I am experiencing right now while drinking my jasmine tea. I can also recognize myself in a mirror and reflect aloud, "This face is mine." Hence one convenient way to define the notion of prereflective self-awareness is by contrasting it with reflective self-awareness. The latter occurs, for instance, whenever one reflectively introspects one's ongoing experiences or during explicit self-recognition of one's face in the mirror. By contrast, prereflective self-awareness does not involve any form of high-order selfmonitoring. One can get a bearing on this phenomenological take on self-awareness by contrasting it with the view of perceptual awareness defended by Brentano. In Brentano's view ([1874] 1973), when I perceive a cat, I am aware that $I$ am perceiving a cat. Importantly, he acknowledges that I do not have here two distinct mental states, but rather one single mental phenomenon: my awareness of the cat is one and the same as my awareness of perceiving it. But by means of this unified mental state, I have an awareness of two objects: the cat and my perceptual experience. Opposing this view, several contemporary philosophers (Legrand 2006; Zahavi 2014) insist on the phenomenological insight according to which my awareness of my experience is not an awareness of it as an object, in the sense that I cannot endorse the perspective of an external observer or spectator on it. In prereflective self-awareness, experience is given not as an object but as a fundamentally first-personal subjective experience. Clearly much more needs to be said about perceptual awareness in general and the related debates in contemporary philosophy of mind, but for the purposes of this chapter, we will restrict our focus to the phenomenological insight according to which our experiences always involve a kind of implicit, prereflective self-awareness that is a more basic form of selfawareness.

This idea has been the focus of much recent empirical research, including investigations that use experimental "tricks" to systematically manipulate sensorimotor signals, promote their integration, or generate conflicts and illusions, and hence study their role in body awareness (for a review, see Blanke, Slater, and Serino 2015). These studies, as well as investigations in neuropsychiatry (for a review, see Jenkinson and Fotopoulou 2014), suggest that primary sensorimotor signals are integrated and organized at different levels of the neurocognitive hierarchy to form several neurocognitively distinct dimensions of minimal, as well as "extended," selfhood. "Body ownership" (the prereflective sense or metacognitive judgment that I am the subject of a voluntary or involuntary movement, or that I am experiencing a certain sensation like touch) and "body agency" (the prereflective sense or metacognitive 
judgment that I am the cause of a movement and its consequences) (Gallagher 2003; Legrand 2006) are related notions here.

Recently, one of the authors of this chapter (AF) has used an influential theory from computational neuroscience, the Free Energy Framework (Friston 2010), to describe the processes that constitute the minimal self as the "mentalization" of sensorimotor signals (Fotopoulou 2015). Although the term "mentalization" is traditionally used in psychology to refer to our cognitive ability to infer the mental states of ourselves and others, its alterna- tive use in this context is deliberate: it aims to ground this traditional concept in its embod-ied origin and highlight that self-awareness is not some "add-on" inferential process of "mind reading" but rather a more fundamental process of organization and schematization of bodily signals that directly and necessarily extends to the mentalization of any-body (see hereafter). While a description of the Free Energy Framework itself goes beyond the scope of the current chapter, we heuristically define "body mentalization" here as the process by which primary sensorimotor signals are progressively integrated and schematized to form multiple models of our embodied states in given environments. These models are under- stood not as static body representations in the brain (e.g., "body schema" versus "body image") but as "hypothetical" (probabilistic, inferential), dynamic, and generative processes (they are constantly updated against received error signals). While we cannot do justice to this topic here, we focus in the next section on how mentalization takes place in relation to different bodily signals derived from the individual body, as well as from other bodies in physical proximity. In doing so, we also position this process in a conceptual space that we think is currently occupied by an arbitrary gulf created between experiential minimalism and social constructivism.

\section{The Mentalization of the Body and Others: The Terrain between Experiential Minimalism and Social Constructivism}

Adopting the view that minimal selfhood emerges from the progressive mentalization of the experience of an active and situated living body within a wider physical and social environment presents us with the crucial question of the role of others in shaping minimal selfawareness. In other words, can we characterize "mineness" and the minimal selfhood without any contrasting others? We address this question by first considering the existing neurophilosophical literature, as recently summarized by Zahavi (2014, 2015a, 2015b). On the one hand, according to the experiential minimalism approach (EM), our experiential life is from the beginning characterized by prereflective self-awareness and by its first-personal character or "mineness," which is an innate, ongoing, and more primary form of self-awareness (see also Gallagher 2005; Legrand 2006; Thompson 2007). On the other hand, according to social constructivism (SC), the self is a socioculturally determined acquisition, emerging in the process of social exchanges and mutual interactions. To put it provocatively, one cannot be a self on one's own but only together with others. 


\section{PROPERTY OF THE MIT PRESS \\ FOR PROOFREADING, INDEXING, AND PROMOTIONAL PURPOSES ONLY}

The Touched Self

Both EM and SC face criticisms. Critics of EM have pointed out that a minimal understanding of selfhood overlooks the crucial role of the open-ended construction of individuality via narratives and language (Gallagher and Hutto 2008). Indeed, given that we are never cut off from the world, who we are crucially depends on the story we and others tell about ourselves. Critics of SC have argued that selfhood cannot be reduced to that which is narrated, and the very mineness of experience is not constitutively dependent on social interactions and intersubjectivity:

I am not disputing that we de facto live together with others in a public world from the very start, but I would deny that the very mineness or for-me-ness of experience is constitutively dependent upon social interaction. In short, I am not disputing the de facto co-existence and co-emergence of experi- ential selfhood and intersubjectivity, but am rather denying their constitutive interdependence. (Zahavi 2015a, 148)

There is much controversy in contemporary social cognition literature, including in the recently formed field of "second-person neuroscience" (Schilbach et al. 2013), over the appropriate understanding of the so-called we-experiences in terms of "we-, shared-, or collective intentionality," "second-person cognition," or "plural self-awareness," and whether these we-experiences precede or presuppose the self-other distinction (Searle 1990; Reddy 2008; Gallotti and Frith 2013; Tuomela 2013; Schmid 2014). For our restricted purposes here, we will focus on the issue of the constitutive interdependency between minimal experiential selfhood and intersubjectivity from a developmental perspective. For example, Zahavi and Rochat (2015) have drawn on phenomenological insights and developmental studies to support the idea that we-experiences are not prior to, or equiprimordial with, self-experiences. What is primordial is the first-personal presence, the mineness that amounts to a primitive and minimal form of selfhood. We agree with Zahavi and Rochat's claim that this basic experiential ownership functions as a precondition for all normative, narrative, and culturally embedded self-interpretations that might occur later in the development. However, we believe that in dismissing the constitutive interdependence of experiential selfhood and intersubjectivity, one runs the risk of throwing the proverbial baby with the bathwater, if intersubjectivity is understood only as the sense of dynamic interactions between two (or more) socially constructed selves. Indeed, a closer look at the development of experiential life at the most primitive levels might reveal the presence of even more primitive forms of embodied relatedness and proximal intersubjectivity, which do not need to posit socially mediated and culturally constructed selves.

In the next sections, we suggest that it is preferable to refocus the very notion of "intersubjectivity" to take into account more basic and proximal forms of embodiment present in early infancy. We begin with some conceptual clarifications regarding the term "perceptual experience" itself, as well as the "observability" condition that shapes these debates, to argue that many accounts tacitly endorse a visuospatial model of perceptual experience, and that this might be misleading in understanding more basic forms of intersubjectivity. By contrast, 


\section{PROPERTY OF THE MIT PRESS \\ FOR PROOFREADING, INDEXING, AND PROMOTIONAL PURPOSES ONLY}

we argue that an understanding of other minds is based on a more direct experience of their bodies as proximal sources of sensory signals, encountered in different conditions of congruency and incongruency with other sensorimotor signals originating from within, on, and outside the physical boundaries of the body. It is the regularities, as well as the unavoidable irregularities, of such processes that determine the progressive mentalization of one's own body and the bodies of others, and therefore the progressive sophistication of the self-other distinction. This view, which avoids an unnecessary prioritization of a "detached-visual" model of perception, is best exemplified by considering the role of interoception and particularly affective touch in infancy and beyond.

\section{Proximal Intersubjectivity: Against a Detached Perspective}

Contemporary debates in philosophy of mind and cognition conceal deep disagreements in the definition of perceptual awareness. To give a full and detailed account of these debates, as fascinating as they are, would require a substantial digression. For our purposes here, we can simply build on the observation that perceptual experience is taken to be the fundamen- tal point of contact with the world, and as such it provides the primary basis on which beliefs, concepts, and knowledge may be formed to relate to the environment. Typically, the relation subject-object constitutes a paradigmatic structured relation of a perceptual experi- ence, where the experiencing subject relates to an object there. For example, Crane and French (2015) note that "perceptual experience, in its character, involves the presentation (as) of ordinary mind-independent objects to a subject, and such objects are experienced as present or there such that the character of experience is immediately responsive to the charac- ter of its objects." An important correlate of the problem of perceptual experience concerns the subject-subject relation, namely, the social perception of other people. Crucially, this model tacitly presupposes a visuospatial perspective of perceptual experience where a subject faces an object from a safe distance there (as opposed to here) and in a detached manner (nocontact). ${ }^{2}$

For example, it is common to claim that the view others have of the infant functions as a social "mirror" through which the child becomes aware of herself. However, as we shall shortly see, this idea of one's "visibility" through others' perceptual awareness and the related typical expression-to see oneself through the eyes of others-is highly misleading when examining more primitive forms of prereflective self-awareness (Ciaunica 2015). For example, Sartre famously argued that my primary experience of the other is an experience that involves my own self-consciousness, that is, a self-consciousness in which I am prereflectively aware that I am a visible object for another. Sartre characterizes my being-for-others as an external dimension of being, and he speaks of the existential alienation provoked by my

2. This discussion has obvious implications for spatial cognition and notions of peripersonal and extrapersonal space that sadly we cannot address within the space limitations of this chapter. 


\section{PROPERTY OF THE MIT PRESS \\ FOR PROOFREADING, INDEXING, AND PROMOTIONAL PURPOSES ONLY}

The Touched Self

encounter with the other (Sartre [1943] 1956, 287). The primary experience of the other is not that I perceive her as some kind of object in which I must encounter a person. Rather, I perceive the other as a subject who perceives me as a visible object. However, recent developmental studies suggest that this type of self-apprehension through others' eyes (based on a visuospatial model of perspective taking) is not the most primitive form of self-other relatedness (Moll and Kadipasaoglu 2013). In the remainder of the chapter, we focus on interoception and touch as perceptually proximal ways to relate to others, and we provide empirical evidence suggesting that at the basic level, self-other relatedness is not primarily experienced in this detached visuospatial fashion.

\section{The Touched Self: The Mentalization of Proximal Bodies}

Questions regarding minimal selfhood and otherness have also received increasing empirical attention in developmental psychology. The results of infant imitation experiments, for instance, have yielded a plethora of interpretations and debates. Other empirical researchers have focused on multisensory integration and other "contingency detection" paradigms (for a review, see Gergely and Watson 1999). For instance, some studies have now illustrated that infants as young as three to five months show sensitivity to body-related, proprioceptivevisual synchrony and, as motor control develops, also spatial congruency (Rochat and Morgan 1995). In such paradigms, infants tend to respond differentially to experimentally controlled and visually presented feedback of their body parts (e.g., their legs) moving synchronously and in spatial congruency to their own movements, rather than manipulated visual feedback that does not have these properties (e.g., asynchronous or incongruent movements). A recent study has further found that newborns detect visual-tactile synchrony in stimuli directed to their own faces and are able to discriminate synchrony from visual-tactile asynchrony (Filippetti et al. 2013). Indeed, the detection of "amodal" properties like synchrony is considered key to the integration of the senses and the organization of perceptual input into distinct, unitary multimodal schemata, a process we have termed "sensorimotor mentalization" in this chapter. This is in fact the basis of most multisensory integration paradigms in adults: sensitivity to synchrony (the so-called glue of the senses) across sensory input allows perceiving subjects to experience unitary multimodal events and to separate stimulation originating from the self and stimulation arising from others. Accordingly, developmental studies on sensitivity to synchrony have been considered as evidence for the early ability for a rudimentary distinction between self and other. This conclusion seems to favor experiential minimalism (EM), in the sense that such a distinction seems to precede the need for intersubjectivity in the constitution of the self.

However, we believe this approach misses an important dimension of such integration and mentalization processes. Indeed, human infants seem to respond to fundamental rules of information organization; however, in their everyday experiences (and in some experimental settings) such amodal rules apply also to information received from other bodies in 


\section{PROPERTY OF THE MIT PRESS FOR PROOFREADING, INDEXING, AND PROMOTIONAL PURPOSES ONLY}

their proximity. Put crudely, the bodies of human caregivers provide an almost continuous embodied engagement in infancy, during which rich patterns of synchrony and asyn- chrony, and other forms of on-off sensory and spatial contingency and congruency, are implemented through a rich repertoire of culturally defined practices of interaction (e.g., hugging, kissing, singing, clapping, stroking, rocking, holding), as well as necessary and frequent routines of embodied engagement required to satisfy the infant's basic biological and psychological needs (e.g., breast-feeding, washing, rubbing-cleaning, skin-to-skin sleep- ing, body-to-body temperature regulation, and skin hydration; see also the following sec- tions). Several volumes have recently focused on visual signals from other bodies, like the mirroring assumptions and theories of different kinds (for a review, see Gallese 2013). How- ever, we propose here that the early, crucial role of such practices in the formation of the minimal self is most obvious when one considers the special case of interpersonal "touch," even in experimental settings. In the aforementioned study of Filippetti and colleagues (2013), for instance, part of the sensory input (the tactile stimulation) was caused by another individual in both the synchronous and the asynchronous conditions. Therefore what determines the early mentalization of one's own body, as opposed to that of another individual, may somewhat paradoxically be caused by social interactions (cosubjectivities). For instance, feeding, sleeping, calming-down, or entertaining routines typically include endless repetitions of multisensory bundles from at least two bodies (e.g., active and passive touch, proprioceptive and vestibular information, smell, temperature, visual and auditory feedback). During such experiences, the infant is therefore responding to regularities and irregularities between the various sensorimotor "bundles" and thus mentalizing its own body in interaction with that of the caregiver. In this sense, the very first-person experience of my body as mine is constituted by the presence of, and interaction with, other bodies in proximity.

Before we go on to further explain why physical contact and interpersonal touch have a unique, primary role in the minimal self, it is necessary to stress that contrary to SC, the critical variable emphasized here is "other interacting bodies," rather than "other minds." While the behavior of caregivers is determined by their feelings and intentions toward infants, such general mental states are not regarded as the critical element of the role of proximal intersubjectivity in the formation of minimal selfhood. We illustrate this point in reference to a recent empirical study on infant holding. Most parents would recognize that it is far easier to calm and put to sleep a crying baby while standing up and walking around the room with the baby in one's arms than by holding the baby in one's arms while seated. Indeed, a recent study of infants younger than six months found that being held and carried by a walking mother led the infants to immediately stop voluntary movement and crying and exhibit a rapid heart rate decrease, compared with holding by a sitting mother (Esposito et al. 2013). Furthermore, similar motor, vocal, and heart rate "calming" responses were observed in mouse pups, supporting the idea of a conserved embodied component of mammalian mother-infant interaction. It should be obvious from 


\section{PROPERTY OF THE MIT PRESS \\ FOR PROOFREADING, INDEXING, AND PROMOTIONAL PURPOSES ONLY}

The Touched Self

these findings that such mechanisms relate to the importance of embodied caregiver-infant interactions per se (e.g., mobile versus static holding), without the need to refer to the sharing of any high-order, mental, or even spatial concepts such as intentionality, empathy, or perspective.

Similar effects of embodied and primarily tactile interactions between parents and their offspring has long been established in other mammals (Harlow 1958; Panksepp 1998). For example, early postnatal maternal tactile stimulation (linking/grooming) has been shown to modify the known adverse effects of prenatal stress on physiological and emotional reactivity later in life (e.g., Vallee et al. 1999). Unfortunately, relevant systematic research in human infants is sparse (Sharp et al. 2012), although touch has long been prioritized by proponents of "kangaroo care" and similar "skin-to-skin" and "touch-based" approaches to parenting and health care (for a review, see Field 2001). However, for the most part, scholars of human infancy tend to claim that such effects in humans are mediated by parents' mental states and related higher-order psychological concepts (e.g., theory of mind, attachment style). Even in theories that have stressed embodied aspects of the infant-caregiver relationship, for example, "affect attunements" (Stern 1985) or "contingent marked mirroring" (Gergely and Watson 1999), these are quickly embedded in more complex mentalistic conceptualizations of the caregiver's mind and therefore the view that infants' minds are first "read out" by moth- ers and then responded to accordingly. While we do not deny the role of such forms of relatedness and intersubjectivity, we agree with Zahavi and others in understanding such factors as secondary to more primitive forms of subjectivity and selfhood. Contrary to extreme versions of experiential minimalism, however, we propose that proximal embodied interactions of caregiving entail a more fundamental form of embodied intersubjectivity that contributes to the constitution of the self from the outset. In the example of the study by Esposito et al. (2013), we assume that the mental states and "mind-reading" capacities of mothers toward their babies do not typically vary depending on whether they are walking or sitting, and yet the particular embodied interactions between mother and infant seem to have direct behavioral and physiological effects on infants. We further specify some of the reasons why such embodied interactions are especially relevant and necessary for the formation of minimal selfhood.

\section{The Felt Self: The Mentalization of Interoceptive Signals}

What kind of bodily signals become "mentalized" to form the basis of minimal selfhood? In empirical research, although scientists have long proposed that bodily self-consciousness relies on an integrated representation of multiple streams of sensory and motor information, there has been a strong bias in the kind of bodily signals studied in this respect. Specifically, most investigations have focused on "multisensory integration" paradigms that study the integration of exteroceptive (e.g., vision, audition, touch) signals, or on sensorimotor integration paradigms that may also include motor, efferent signals, and proprioceptive or 


\section{PROPERTY OF THE MIT PRESS \\ FOR PROOFREADING, INDEXING, AND PROMOTIONAL PURPOSES ONLY}

vestibular feedback. Remarkably, however, until recently little work on bodily self-awareness concerned interoception. Interoception refers to the perception of the physiological condition of the body, involving representations from multiple modalities such as temperature, itch, pain, cardiac signals, respiration, hunger, thirst, pleasure from sensual touch, and other bodily feelings. It is distinct from the exteroceptive system, which refers to the classical sensory modalities for perceiving the external environment, as well as proprioceptive, vestibular, and kinesthetic input about the movement and location of the body in space (Blanke and Metzinger 2009; Craig 2002; Critchley et al. 2004). Crucially, contrary to classic views of interoception as "the perception of the body from within," the current notion of interoception is tightly linked to homeostasis and emotion. Interoceptive signals are considered crucial in informing the organism about the homeostatic state of the body in relationship to experiences originating both from within the organism (e.g., cardiac and respiratory functions, digestion, hunger, thirst) and from outside it (e.g., taste, smell, affective touch, pain). Accordingly, interoception is thought to rely on separate specialized neuroanatomical sys tems that are associated with the autonomic nervous system, special spinal cord pathways, and subcortical and cortical brain areas mapping motivational and homeostatic states (Craig 2009; Critchley et al. 2004; Damasio 1994; Panksepp 1998).

A number of researchers have recently argued that interoception is uniquely related to the generation of subjective feelings, informing the organism about its levels of arousal, bodily needs, and the value or valence of stimuli. As such, interoception has been ascribed a central role in the core of self-awareness (Craig 2009; Critchley et al. 2004; Damasio 1994; Seth, Barrett, and Barnett 2011). Important for our purposes here, preliminary evidence also suggests that interoception can uniquely shape the minimal self, as studied in multisensory integration paradigms and neuropsychiatric disorders (for a review, see Seth 2013). For example, participants with lower abilities to detect their own heartbeat seem more susceptible to bodily illusions of synchronous visuotactile stimulation (Tajadura-Jiménez, Longo, Coleman, and Tsakiris, 2012).

How can one reconcile this view with more classical considerations of the constitution of the minimal self and related debates on intersubjectivity as outlined earlier? At first sight, the potential role of interoception in the minimal self may be interpreted as evidence in favor of experiential minimalism. Specifically, one could say that the mentalization of the body and the constitution of the minimal self are mediated by an innate, specialized system that informs the organism about the homeostatic state of the body and particularly of sensations arising from within the organism. The resulting inner feelings of "arousal," "wakefulness," and "wellness," or lack thereof, combined with exteroceptive and motor signals regarding the body, could thus form the basis of subjectivity and the self, and a fundamental source of information regarding the self-other distinction. This is indeed the view that several scientists and scholars have recently put forward (Craig 2009; Damasio 1994; Critchley et al. 2004; Seth 2013). On closer inspection, however, interoception and its properties point to a view that regards intersubjectivity as fundamental and necessary in shaping the mentalization of 


\section{PROPERTY OF THE MIT PRESS \\ FOR PROOFREADING, INDEXING, AND PROMOTIONAL PURPOSES ONLY}

The Touched Self

interoception and not the other way around. The latter claim is supported by two main observations that we unpack in the following two sections, respectively: (a) interoception itself is derived from the outside and other bodies as much as from the inside of the body; and (b) in early infancy, when the motor system is not yet developed, the functioning of several interoceptive modalities depends wholly on embodied interactions with other bodies.

\section{The Affectively Touched Self: The Pleasure of Proximal Interactions}

As previously discussed, contrary to classical views, contemporary accounts have defined interoception as the set of modalities that inform the organism about the homeostatic state of the body in relationship to experiences originating from within the organism (e.g., car- diac awareness, hunger) or outside it (e.g., taste, smell, affective touch, pain). We use the example of affective touch to illustrate the importance of this reclassification (for similar considerations and findings on the domain of pain, see Krahé et al. 2013, 2015; Decety and Fotopoulou 2014). Indeed, recent neurophysiological, neuroimaging, and behavioral studies suggest that certain single tactile experiences on the skin, such as the reception of gentle, caress-like strokes, are processed by two partly independent neurocognitive systems. As has been known for decades, tactile stimuli are processed in terms of their exteroceptive, discriminatory processes in classical peripheral pathways and somatosensory cortical areas. Recent research has demonstrated, however, that a specialized peripheral and central system seems to code for the affective properties of the same tactile stimulus. Contrary to purely sensory touch, composed of skin mechanoreceptors projecting to the thalamus and primary somatosensory cortex, the neurophysiological system for affective touch (Vallbo, Olausson, and Wessberg 1999) seems to rely on a distinct subgroup of mechanoreceptors, tactile Cfibers, responding only to slow (between 1 and $10 \mathrm{~cm} / \mathrm{s}$ ), caress-like touch and leading to subjective pleasantness (Löken et al. 2009). Crucially, C-tactile afferents take a distinct ascending pathway from the periphery to a different part of the thalamus and then to the posterior insular cortex (Morrison, Bjornsdotter, and Olausson 2011). According to some researchers, the latter pathway is considered as mediating an early convergence of sensory and affective signals about the body, which are then re-represented in the mid and anterior insula, the proposed sites of interoceptive awareness (Craig 2009; Critchley et al. 2004).

Thus affective touch seems to simultaneously capture information about the "inner" body (e.g., "this experience feels good or not") and the external world (e.g., "this is a material with little friction, moving slowly"). Crucially, a recent study found that nine-month-old infants are sensitive to the particular physical properties of affective touch, in the sense that CT- optimal but not nonoptimal velocities of tactile stimulation led to heart rate deceleration in the infants, possibly reflecting relaxation and increases in their behavioral engagement (gaze shifts and duration of looks) with the stroking stimulus. We speculate that this unique 


\section{PROPERTY OF THE MIT PRESS \\ FOR PROOFREADING, INDEXING, AND PROMOTIONAL PURPOSES ONLY}

parallel (by definition, synchronous and spatially congruent) activation of pathways relating to the internal representation of the body, as well as the external, mostly social world of the infant (see the previous section regarding the presence of social touch in infancy), presumably acts as an early developmental source not only of self-other relatedness and social connection (for the so-called social touch hypothesis, see Morrison, Loken, and Olausson 2010) but also of bodily information regarding the self-other distinction. Paradoxically, given its dual sensory-discriminatory and affective-motivational nature, social touch, an essential part of early mother-infant interactions, may have a unique developmental role in progressively establishing the physical boundaries of the psychological self. Unfortunately, to our knowledge, no systematic developmental studies have focused specifically on the role of affective touch in the formation of the minimal self. However, the aforementioned application of multisensory integration paradigms to the study of infant body perception (e.g., Filippetti et al. 2013) suggests that the specific developmental role of affective social touch can soon be studied in early infancy and childhood.

In addition, indirect confirmation of our suggestion comes from studies on adults. Recent research has shown that the perception of affective touch can provide information about the emotions and thoughts of other individuals, that is, the touch providers (Herten-stein et al. 2006) and the touch receivers (Gentsch, Panagiotopoulou, and Fotopoulou 2015). More specifically as regards the minimal self, a series of recent studies focused on the role of affective touch in the sense of body ownership. The rubber hand illusion is a para- digm involving the illusion of ownership of a foreign hand following synchronous visuo- tactile stimulation between one's own unseen hand and another hand seen in proximity and in a congruent spatial position to one's real hand. Using this paradigm, three indepen- dent studies, one of them from AF's lab, have now found that slow, caress-like touch of CT-optimal velocities and properties enhanced various subjective and behavioral measures of the rubber hand illusion more than fast, emotionally neutral touch (Crucianelli et al. 2013; Lloyd et al. 2013; van Stralen et al. 2014). That is, affective, pleasant touch delivered by another individual seems to play a unique role in the process of multisensory integra- tion that determines how a body part is subjectively experienced as mine. Support for this idea can also be found in several studies on patients with clinical disorders of the minimal self, such as patients with body ownership disturbances, who seem able to at least momen- tarily accept their disowned arm as theirs following affective touch (for a review, see Gentsch et al., forthcoming).

\section{When the Motor System Is Not Yet Developed: The Social Mentalization of the Body}

Young infants show considerable movement of the head, including the face and eyes, the limbs and the trunk, especially when in certain neurophysiological states. Nevertheless they lack strength and control in their large antigravity muscles and are helpless in supporting their own weight, and they are unable to initiate and execute complex sequences of 


\section{PROPERTY OF THE MIT PRESS \\ FOR PROOFREADING, INDEXING, AND PROMOTIONAL PURPOSES ONLY}

The Touched Self

purposeful movements. Therefore a young infant cannot position and balance itself, feed itself, thermoregulate, or protect itself from tissue damage (e.g., skin burns, bone fractures, etc.). Freud wrongly assumed that this human need for early nurturance and care by conspecifics is the ultimate motivation for our early social relating. Seminal studies have since established that humans have developed an innate social attachment drive, unrelated to hunger or thermoregulation, and a corresponding lifelong need for social connection (Bowlby 1969; Harlow 1958; Panksepp 1998). Contrary, however, to recent emphasis on mentalistic concepts such as "attachment styles," it is useful to remember that proponents of this view have indeed emphasized the embodied rather than the mentalistic dimensions of this drive, such as the "need for physical proximity" (Bowlby 1969), "contact comfort" (Harlow 1958), and tickling or bodily play (Panksepp 1998). Indeed, we believe that the primacy of our social attachment drive should not obscure the important embodied role of caregivers in regulating the infant's interoceptive states and in turn the foundations of the minimal self. Thus, contrary to current "mentalistic" views of self-formation, we suggest that the origins of the mentalization process itself are not only embodied (as outlined in the previous sections) but also by necessity involve other people's bodies.

Specifically, in the previous sections, we have described as "the mentalization of the body" the process of detecting "amodal" properties like synchrony between the senses and organizing sensory input of both personal and interpersonal origins into distinct, unitary multimodal schemata. We have also stressed that such senses refer not only to exteroception but also to interoception, the senses that inform the organism about the homeostatic state of the body. Moreover, the mentalization of the body involves not only perceptual integra- tion and subsequent inferences but also sensorimotor integration (active inference, in the terminology of the Free Energy Framework). Given the infant's immature motor system, however, what kind of models can she form? The rudimentary motor system of the infant affords several opportunities for her to learn and build generative models in her first unaided sensorimotor interactions with the environment. For instance, an infant can learn that closing her eyes or looking away causes changes in her visual input, which she can then learn to implement when sudden large changes in environmental light occur. In the case of sev- eral interoceptive modalities, however, no movement on the part of the infant alone can change her interoceptive state. In the terms of the Free Energy Framework, there can be no prediction errors, or learning in the longer-term, on the basis of active inference.

In this section, we put forward the radical claim that it is exactly the fact that human infants are born without a fully mature motor system that can change its own physiological states that determines the constitutional role of proximal intersubjectivity in minimal selfhood. As infants experience physiological changes as both internal and external conditions, they can engage in reflexive autonomic and motor behaviors (e.g., crying, kicking, exploring, sucking, etc.). These active behaviors, however, return only rudimentary sensory feedback that rarely changes the interoceptive state of the infant (e.g., the infant can quickly learn to perceive its own crying or can start to associate kicking with the tactile input encountered if 


\section{PROPERTY OF THE MIT PRESS FOR PROOFREADING, INDEXING, AND PROMOTIONAL PURPOSES ONLY}

the legs touch something in the environment, but these experiences and newly acquired schemata will not change his levels of arousal or satiation). Instead, in good-enough caregiving environments, such behaviors are met-not only by facial expressions and other aforementioned mentally "attuned" responses, but also crucially with a variety of proximal, embodied responses, such as soothing touch, holding, feeding, and so on, as described earlier-and can produce changes in the infant's behavior, physiology, and particularly her interoceptive state (e.g., heart rate reductions, satiation, etc.). It follows that feelings of bodily satisfaction, pain, and pleasure, and the lack thereof, are primarily constituted as "mine" only via behaviors that engage the interacting other (see also Gergely and Watson 1999), rather than being fundamental processes of the singular individual and its body, as certain theories assume (e.g., Damasio 2010; Craig 2009).

Two further points are needed to clarify this position. First, the infant's behaviors during proximal interactions (e.g., crying) are not the manifestation of a preexisting set of psychologically differentiated "feelings" or "subjective states," as the experiential minimalism view implies. Rather, we propose that the infant experiences changes in mentally "undifferentiated" states of physiological arousal, or alertness, as well as "sEEking" in the active domain (SEEKING is conceived as a primitive motivational system in neuroscience, linked with the neurobiology of the neurotransmitter dopamine and described as an "objectless" urge for action toward the environment, an active version of the notion of arousal; see Panksepp 1998). The infant progressively learns to associate such physiological states with particular behaviors and responses (and later with language and cultural responses; indeed, culture is present in the minimal self only as embodied practices between people, as explained earlier). For instance, crying no longer is only associated with the initial state of physiological change but also predicts a set of external behaviors, some of which in turn are anticipated to change the initial state, and the overall process "binds" physiology to subjectively experienced states such as "pain" or "unpleasure." We believe this is the embodied basis of subjective "feeling states," which therefore do not preexist embodied encounters. The more rudimentary dimensions of arousal and SEEKING are, in our view, the only aspects of subjectivity that can be put forward in support of experiential minimalism. However, as they do not require any preexisting perspectival notion, or self-other distinction (e.g., there is no reason to assume that changes in physiological arousal are experienced by an infant in a way that is anchored to any notion of a self, i.e., as arousal experienced by me), it is unclear whether they should be seen as the basis of minimal selfhood.

Finally, our position does not assume that the infant mentalizes its own body and thus develops a minimal self, because the adult is able to correctly "read out" the infant's psychological states and "metabolize" them into appropriate emotions and feelings (Fonagy et al. 2004). Successful mind reading (Fonagy et al. 2004; Gallese 2013) or "affective attunement" (Stern 1985) by the adult ensures this process can continue to develop successfully as the child grows, and the interactions indeed need to be "attuned" to a richer and more fixed mental, generative models. However, as we have already stressed (see sec. 3), the critical 


\section{PROPERTY OF THE MIT PRESS \\ FOR PROOFREADING, INDEXING, AND PROMOTIONAL PURPOSES ONLY}

The Touched Self

variables in the initial processes of social mentalization emphasized here are assumed to be bodily and exploratory rather than psychological and fixed. We believe caregivers interact with infants mostly in a dynamic, trial-and-error fashion, trying to discover and learn what each infant needs in each instance and from each caregiver. In the same instance, they are contributing to the infant's self-formation and learning. In simple terms, the self is not only constituted intersubjectively but also constitutes particular emotional intersubjectivities at the same time. If this were so, early care would be far easier than it seems to be, and a good textbook could really tell us what all infants need.

\section{Conclusion}

Is the minimal self already relational in its very bodily foundations? At first sight, it is tempting to agree with Zahavi's claim that "we should not accept being forced to choose between viewing selfhood as either a socially constructed achievement or an innate and culturally invariant as a given. Who we are is both made and found" (2015a, 147). Indeed, the minimalist notion of an experiential self is fully compatible with a more complex notion of a socially and normatively embedded self, and researchers have proposed several ways of framing this relation. However, we have tried to argue here in favor of a more radi- cally embodied view of intersubjectivity itself that cuts across the distinction between innate and socially constructed notions of selfhood. Proximal intersubjectivity itself is not an index of socialization, or the product of cultural practices, but rather a fundamental condition of human survival, supported by strong human attachment instincts. Specifi- cally, we have outlined that particular types of affective touch and more generally physical contact and proximal interactions are crucial for the mentalization of the body and the formation of minimal selfhood. We have also made the further, radical claim that in early infancy, when the motor system is immature, proximal intersubjectivity is necessary for the mentalization of interoceptive states and therefore the corresponding core aspects of the minimal self. There is thus no gap between the minimal and the interactive self-as con- strained and embedded in cultural frameworks-for there is a deep continuity between the principles that govern the mentalization of sensorimotor signals in the singular individual and the mentalization of anybody via physical proximity and interaction. Our approach thus echoes Merleau-Ponty's view according to which one must consider "the relation with others not only as one of the contents of our experience but as an actual structure in its own right” ([1960] 1964, 140; our italics).

\section{Acknowledgments}

Aikaterini Fotopoulou was supported by a European Research Council Starting Investigator Award (ERC-2012-STG GA313755) and a Volkswagen Foundation European Platform for Life Sciences, Mind Sciences, and the Humanities grant. 


\section{PROPERTY OF THE MIT PRESS FOR PROOFREADING, INDEXING, AND PROMOTIONAL PURPOSES ONLY}

Anna Ciaunica was supported by a Foundation for Science and Technology Fellowship Grant (FCT) (SFRH/BPD/94566/2013).

\section{References}

Blanke, O. 2012. Multisensory brain mechanisms of bodily self-consciousness. Nature Reviews: Neuroscience 13:556-571.

Blanke, O., and T. Metzinger. 2009. Full-body illusions and minimal phenomenal selfhood. Trends in Cognitive Sciences 13 (1): 7-13.

Blanke, O., M. Slater, and A. Serino. 2015. Behavioral, neural, and computational principles of bodily self-consciousness. Neuron 88 (1): 145-166.

Bowlby, J. 1969. Attachment and Loss, vol. 1: Attachment. Basic Books.

Brentano, F. [1874] 1973. Psychologyfrom an Empirical Standpoint. Trans. A. C. Rancurello, D. B. Terrell, and L. L. McAlister. Routledge.

Ciaunica, A. 2015. Basic forms of pre-reflective self-consciousness: A developmental perspective. In Pre-reflective Self-Consciousness: Sartre and Contemporary Philosophy of Mind, ed. S. Miguens, G. Preyer, and C. Morando, 422-438. Routledge.

Clark, A. 2013. Whatever next? Predictive brains, situated agents, and the future of cognitive science. Behavioral and Brain Sciences 36 (3): 181-204.

Craig, A. D. 2002. How do you feel? Interoception: The sense of the physiological condition of the body. Nature Reviews: Neuroscience 3:655-666.

Craig, A. D. 2009. How do you feel-now? The anterior insula and human awareness. Nature Reviews: Neuroscience 10:59-70.

Crane, T., and C. French. 2015. The problem of perception. In Stanford Encyclopedia of Philosophy, spring 2016 edition, ed. E. N. Zalta. http://plato.stanford.edu/archives/spr2016/entries/perception-problem.

Critchley, H. D., S. Wiens, P. Rotshtein, A. Öhman, and R. D. Dolan. 2004. Neural systems supporting interoceptive awareness. Nature Neuroscience 7:189-195.

Crucianelli, L., N. K. Metcalf, A. K. Fotopoulou, and P. M. Jenkinson. 2013. Bodily pleasure matters: Velocity of touch modulates body ownership during the rubber hand illusion. Frontiers in Psychology 4:703.

Damasio, A. R. 1994. Descartes'Error: Emotion, Reason, and the Human Brain. G. P. Putnam's Sons.

Damasio, A. R. 2010. Self Comes to Mind: Constructing the Conscious Brain. Heinemann.

Decety, J., and A. Fotopoulou. 2014. Why empathy has a beneficial impact on others in medicine: Unifying theories. Frontiers in Behavioral Neuroscience 8:457. 


\section{PROPERTY OF THE MIT PRESS FOR PROOFREADING, INDEXING, AND PROMOTIONAL PURPOSES ONLY}

The Touched Self

De Jaegher, H., and E. A. Di Paolo. 2007. Participatory sense-making: An enactive approach to social cognition. Phenomenology and the Cognitive Sciences 6:485-507.

Esposito, G., S. Yoshida, R. Ohnishi, Y. Tsuneoka, M. del C. Rostagno, S. Yokota, S. Okabe, et al. 2013. Infant calming responses during maternal carrying in humans and mice. Current Biology 23 (9): 739745. doi:10.1016/j.cub.2013.03.041.

Field, T. 2001. Touch. MIT Press.

Filippetti, M. L., M. H. Johnson, S. Lloyd-Fox, D. Dragovic, and T. Farroni. 2013. Body perception in newborns. Current Biology 23 (23): 2413-2416.

Fonagy, P., G. Gergely, E. L. Jurist, and M. Target. 2004. Affect Regulation, Mentalization, and the Development of Self. Karnac.

Fotopoulou, A. 2015. The virtual bodily self: Mentalisation of the body as revealed in anosognosia for hemiplegia. Consciousness and Cognition 33:500-510.

Friston, K. 2010. The free-energy principle: A unified brain theory. Nature Reviews: Neuroscience 11:127-138.

Gallagher, S. 2000. Philosophical conceptions of the self: Implications for cognitive science. Trends in Cognitive Sciences 4 (1): 14-21.

Gallagher, S. 2003. Bodily self-awareness and object-perception. Theoria et Historia Scientiarum: International Journal for Interdisciplinary Studies 7 (1): 53-68.

Gallagher, S. 2005. How the Body Shapes the Mind. Oxford University Press.

Gallagher, S., and D. Hutto. 2008. Understanding others through primary interaction and narrative practice. In The Shared Mind: Perspectives on Intersubjectivity, ed. J. Zlatev, T. P. Racine, C. Sinha, and E. Itkonen, 17-38. John Benjamins.

Gallese, V. 2013. Mirror neurons, embodied simulation and a second-person approach to mind reading. Cortex 49:2954-2956. doi:10.1016/j.cortex.2013.09.008.

Gallotti, M., and C. Frith. 2013. Social cognition in the we-mode. Trends in Cognitive Sciences 17 (4): $160-165$.

Gentsch, A., E. Panagiotopoulou, and A. Fotopoulou. 2015. Active interpersonal touch gives rise to the social softness illusion. Current Biology 25 (18): 2392-2397.

Gentsch, A., L. Crucianelli, P. Jenkinson, and A. Fotopoulou. Forthcoming. The touched self: Affective touch and body awareness in health and disease. In Affective Touch and the Neurophysiology of CT Afferents, ed. H. Olausson, J. Wessberg, I. Morrison, and F. McGlone. Springer.

Gergely, G., and J. S. Watson. 1999. Early social-emotional development: Contingency perception and the social-biofeedback model. In Early Socialization, ed. P. Rochat, 101-136. Erlbaum.

Harlow, H. F. 1958. The nature of love. American Psychologist 13:673-685. 


\section{PROPERTY OF THE MIT PRESS FOR PROOFREADING, INDEXING, AND PROMOTIONAL PURPOSES ONLY}

Hertenstein, M. J., D. Keltner, B. App, B. A. Bulleit, and A. R. Jaskolka. 2006. Touch communicates distinct emotions. Emotion 6 (3): 528-533. doi:10.1037/1528-3542.6.3.528.

Hohwy, J. 2007. The sense of self in the phenomenology of agency and perception. Psyche 13 (1): 1-20.

Jenkinson, P. M., and A. Fotopoulou. 2014. Understanding Babiski’s anosognosia: 100 years later. Cortex 61:1-4.

Krahé, C., A. Springer, J. A. Weinman, and A. Fotopoulou. 2013. The social modulation of pain: Others as predictive signals of salience-a systematic review. Frontiers in Human Neuroscience 7:386.

Krahé, C., Y. Paloyelis, H. Condon, P. M. Jenkinson, S. C. R. Williams, and A. Fotopoulou. 2015. Attachment style moderates partner presence effects on pain: A laser-evoked potentials study. Social Cognitive and Affective Neuroscience 10:1030-1037.

Legrand, D. 2006. The bodily self: The sensorimotor roots of pre-reflective self-consciousness. Phenomenology and the Cognitive Sciences 5:89-118.

Lloyd, D. M., V. Gillis, E. Lewis, M. J. Farrell, and I. Morrison. 2013. Pleasant touch moderates the subjective but not objective aspects of body perception. Frontiers in Behavioral Neuroscience 7:207.

Löken, L. S., J. Wessberg, I. Morrison, F. McGlone, and H. Olausson. 2009. Coding of pleasant touch by unmyelinated afferents in humans. Nature Neuroscience 12 (5): 547-548.

Menary, R. 2007. Cognitive Integration: Mind and Cognition Unbounded. Palgrave.

Merleau-Ponty, M. [1960] 1964. The child's relations with others. Trans. W. Cobb. In The Primacy of Perception and Other Essays on Phenomenological Psychology, the Philosophy of Art, History, and Politics, ed. J. M. Edie, 96-155. Northwestern University Press.

Metzinger, T. 2003. Being No One: The Self-Model Theory of Subjectivity. MIT Press.

Moll, H., and D. Kadipasaoglu. 2013. The primacy of social over visual perspective-taking. Frontiers in Human Neuroscience 7:558.

Morrison, I., M. Bjornsdotter, and H. Olausson. 2011. Vicarious responses to social touch in posterior insular cortex are tuned to pleasant caressing speeds. Journal of Neuroscience 31 (26): 9554-9562. doi:10.1523/JNEUROSCI.0397-11.2011.

Morrison, I., L. S. Loken, and H. Olausson. 2010. The skin as a social organ. Experimental Brain Research 204 (3): 305-314.

Panksepp, J. 1998. Affective Neuroscience: The Foundations of Human and Animal Emotions. Oxford University Press.

Prinz, W. 2012. Open Minds: The Social Making of Agency and Intentionality. MIT Press.

Reddy, V. 2008. How Infants Know Minds. Harvard University Press.

Rochat, P., and R. Morgan. 1995. Spatial determinants in the perception of self-produced leg movements by 3- to 5-month-old infants. Developmental Psychology 31 (4): 626-636. 


\section{PROPERTY OF THE MIT PRESS FOR PROOFREADING, INDEXING, AND PROMOTIONAL PURPOSES ONLY}

The Touched Self

Sartre, J.-P. [1943] 1956. Being and Nothingness. Trans. H. E. Barnes. Philosophical Library.

Schilbach, L., B. Timmermans, V. Reddy, A. Costall, G. Bente, and T. Schlicht. 2013. Toward a secondperson neuroscience. Behavioral and Brain Sciences 36:393-414.

Schmid, H. B. 2005. Wir-Intentionalität: Kritik des ontologischen Individualismus und Rekonstruktion der Gemeinschaft. Karl Alber.

Schmid, H. B. 2014. Plural self-awareness. Phenomenology and the Cognitive Sciences 13 (1): 7-24.

Searle, J. R. 1990. Collective intentions and actions. In Intentions in Communication, ed. P. R. Cohen, J. Morgan, and M. Pollack, 401-415. MIT Press.

Seth, A. K. 2013. Interoceptive inference, emotion, and the embodied self. Trends in Cognitive Sciences 17 (11): 565-573. doi:10.1016/j.tics.2013.09.007.

Seth, A. K., A. B. Barrett, and L. Barnett. 2011. Causal density and integrated information as measures of conscious level. Philosophical Transactions of the Royal Society A 369:3748-3767.

Sharp, H., A. Pickles, M. Meaney, K. Marshall, F. Tibu, and J. Hill. 2012. Frequency of infant stroking reported by mothers moderates the effect of prenatal depression on infant behavioural and physiological outcomes. PLoS One 7 (10): e45446. doi:10.1371/journal.pone.0045446.

Stern, D. N. 1985. Affect attunement. In Frontiers of Infant Psychiatry, vol. 2, ed. D. Call, E. Galenson, and R. L. Tyson, 3-14. Basic Books.

Tajadura-Jiménez, A., M. Longo, R. Coleman, and M. Tsakiris. 2012. The person in the mirror: Using the enfacement illusion to investigate the experiential structure of self-identification. Consciousness and Cognition 21 (4): 1725-1738.

Thompson, E. 2007. Mind in Life: Biology, Phenomenology, and the Sciences of Mind. Harvard University Press.

Tuomela, R. 2013. Social Ontology: Collective Intentionality and Group Agents. Oxford University Press.

Vallbo, A. B., H. Olausson, and J. Wessberg. 1999. Unmyelinated afferents constitute a second system coding tactile stimuli of the human hairy skin. Journal of Neurophysiology 81 (6): 2753-2763.

Vallee, M., S. MacCari, F. Dellu, H. Simon, M. Le Moal, and W. Mayo. 1999. Long-term effects of prenatal stress and postnatal handling on age-related glucocorticoid secretion and cognitive performance: A longitudinal study in the rat. European Journal of Neuroscience 11 (8): 2906-2916.

Varela, F., E. Thompson, and E. Rosch. 1991. The Embodied Mind: Cognitive Science and Human Experience. MIT Press.

van Stralen, H. E., M. J. van Zandvoort, S. S. Hoppenbrouwers, L. M. Vissers, L. J. Kappelle, and H. C. Dijkerman. 2014. Affective touch modulates the rubber hand illusion. Cognition 131 (1): 147158.

Zahavi, D. 2005. Subjectivity and Selfhood: Investigating the First-Person Perspective. MIT Press. 
Zahavi, D. 2014. Self and Other: Exploring Subjectivity, Empathy, and Shame. Oxford University Press.

Zahavi, D. 2015a. Self and other: From pure ego to co-constituted we. Continental Philosophy Review 48 (2): $143-160$.

Zahavi, D. 2015b. You, me and we: The sharing of emotional experiences. Journal of Consciousness Studies 22 (1-2): 84-101.

Zahavi, D., and P. Rochat. 2015. Empathy $\neq$ sharing: Perspectives from phenomenology and developmental psychology. Consciousness and Cognition 36:543-553. 